\title{
Evaluation of Solver and Time Stepping Options and RIA Demonstration
}

\author{
Russell J Gardner, Charles P Folsom, \\ Wen Jiang, Jason D Hales, Benjamin W \\ Spencer
}

\section{October 2018}

The INL is a U.S. Department of Energy National Laboratory operated by Battelle Energy Alliance

Idaho National Laboratory 


\title{
Evaluation of Solver and Time Stepping Options and RIA Demonstration
}

\author{
Russell J Gardner, Charles P Folsom, Wen Jiang, Jason D Hales, Benjamin W \\ Spencer
}

October 2018

Idaho National Laboratory Idaho Falls, Idaho 83415

http://www.inl.gov

Prepared for the

U.S. Department of Energy

Under DOE Idaho Operations Office

Contract DE-AC07-05ID14517 


\section{Evaluation of Solver} Options and RIA Demonstration

\section{L3:FMC.P17.11}

Russell J. Gardner, INL Charles P. Folsom, INL Wen Jiang, INL Jason D. Hales, INL Benjamin W. Spencer, INL

September 28, 2018
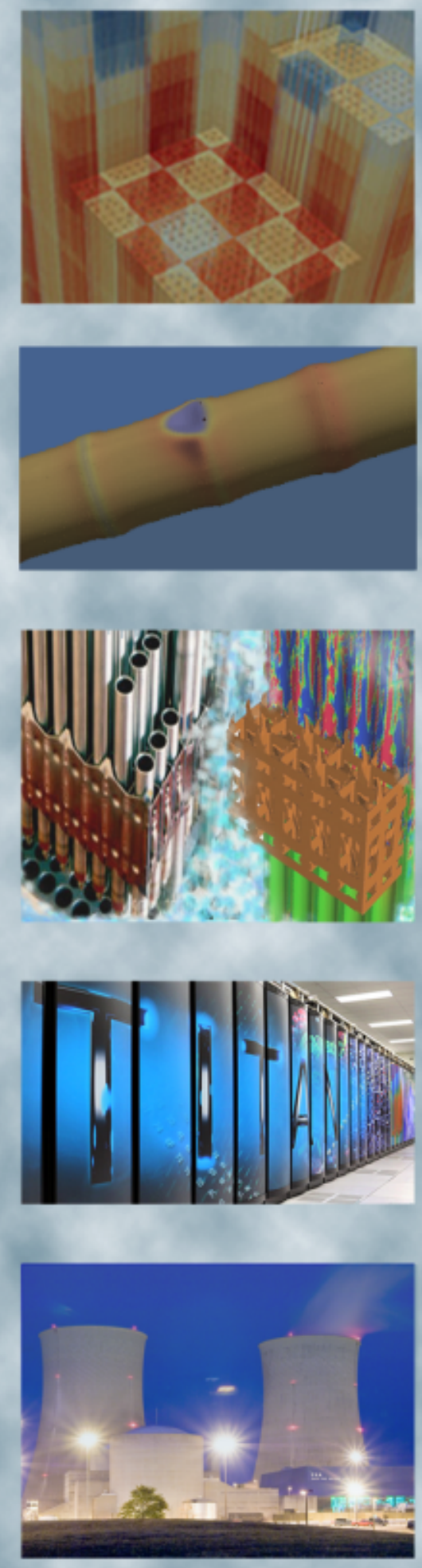
REVISION LOG

\begin{tabular}{|c|c|c|l|}
\hline Revision & Date & Affected Pages & \multicolumn{1}{c|}{ Revision Description } \\
\hline 0 & $09 / 28 / 2018$ & All & Initial Release \\
\hline & & & \\
\hline & & & \\
\hline & & & \\
\hline
\end{tabular}

\section{Document pages that are:}

Export Controlled NONE

IP/Proprietary/NDA Controlled NONE

Sensitive Controlled NONE

This report was prepared as an account of work sponsored by an agency of the United States Government. Neither the United States Government nor any agency thereof, nor any of their employees, makes any warranty, express or implied, or assumes any legal liability or responsibility for the accuracy, completeness, or usefulness of any information, apparatus, product, or process disclosed, or represents that its use would not infringe privately owned rights. Reference herein to any specific commercial product, process, or service by trade name, trademark, manufacturer, or otherwise, does not necessarily constitute or imply its endorsement, recommendation, or favoring by the United States Government or any agency thereof. The views and opinions of authors expressed herein do not necessarily state or reflect those of the United States Government or any agency thereof.

\section{Requested Distribution:}

To: FMC FA Lead

Copy: CASL PM 


\title{
Evaluation of Solver Options and RIA Demonstration
}

\author{
Russell J. Gardner ${ }^{1}$, Charles P. Folsom ${ }^{2}$, Wen Jiang ${ }^{1}$, Jason D. Hales ${ }^{1}$, Benjamin W. Spencer ${ }^{1}$ \\ ${ }^{1}$ Fuel Modeling and Simulation \\ ${ }^{2}$ Experiment Analysis \\ Idaho National Laboratory \\ P.O. Box 1625 \\ Idaho Falls, ID 83415-3840
}

September 28, 2018

\section{Introduction}

This report summarizes Bison development and application in three areas:

- Development of the layered 1D approach to permit its use with the reference residual convergence criterion that is widely used for 2D and 3D Bison simulations. For higher-dimensional simulations, this approach has resulted in significant improvements in robustness and solution efficiency, and it is expected to result in similar benefits for $1 \mathrm{D}$ simulations of light water reactor (LWR) rods.

- A study on the effects of the chosen convergence tolerances on solution difficulty and accuracy was performed for a prototypical layered 1D simulation of an LWR fuel rod. The effect of convergence tolerance on iteration counts, run times, and several quantities of interest in the solution are compared for the various convergence tolerance. This study will be helpful for guiding the choice of convergence tolerances.

- A demonstration of a reactivity insertion accident (RIA) simulation with Bison within the coupled VERA code was performed. The MPACT neutron transport code and the CTF thermal-hydraulic code provided inputs to Bison for a postulated control rod ejection scenario. This demonstrates the ability of the VERA environment to consider coupling effects not only for normal operation, but for accident conditions. 


\section{Reference Residual Approach for Layered 1D Simulations}

Selecting an appropriate convergence criterion is extremely important for ensuring robust and accurate behavior of any nonlinear physics solver. In MOOSE-based applications such as Bison, the nonlinear solver iteratively modifies the solution vector for the entire coupled system, and considers the problem converged when the residual is below an acceptable tolerance. MOOSE allows the user to specify a relative and and absolute tolerance, and the solution is considered converged when either the relative or absolute criterion is reached.

By default, MOOSE takes the $L^{2}$ norm of the residual vector, and compares that to the initial value of that residual for the current time to check for relative convergence, and directly considers the $L^{2}$ norm of the residual for absolute convergence. This default relative convergence check has proven problematic for Bison models, which often have the same power applied for many steps during steady state operation. When the loading conditions are roughly constant, the solution from the previous step is close to the converged solution for the current step, so the initial residual can be very low, which forces the solver to converge to an unreasonably tight tolerance.

To address this problem, an alternative approach, implemented within the ReferenceResidualProblem class in MOOSE, was implemented several years ago, and has been used quite successfully on 2D and 3D models. This approach checks for convergence by comparing the residual to a user-controllable reference quantity. The reference quantity is specified in the form of a set of AuxVariables that contain physically meaningful quantities. The convergence criterion used in ReferenceResidualProblem checks convergence of the solution variables individually. When the $L^{2}$ norm of the residual for each solution variable is less than either the relative tolerance times the $L^{2}$ norm of the corresponding reference variable or the absolute tolerance, the solution is considered converged.

The Layered 1D capability in Bison has proven to be a useful technique for efficiently modeling the global response of light water reactor (LWR) fuel rods. Because the Layered 1D capability employs scalar variables to represent the out-of-plane strain, it did not initially work with ReferenceResidualProblem. The present effort performed needed development for these two to work together. The components of this capability are described below.

\subsection{Computation of Reference Quantities for Scalar Variables}

The reference quantity typically used for field variables is a vector of the conjugate to the solution variable for all nodes with boundary conditions. For mechanics problems, this is a vector of reaction forces, while for heat transfer or diffusion problems, this is an integrated flux. This is typically computed by using the save_in option in MOOSE within the kernels to instruct the kernel to assemble its quantity to an auxiliary variable. When the model is converged, this auxiliary variable contains values that are approximately zero at all interior nodes because the contributions from the connected elements balance each other out, but is nonzero on boundaries with prescribed conditions.

A similar process was needed to compute meaningful reference quantities for convergence checking for scalar variables. The generalized plane system computes the residual for the scalar out of plane strain as the integral of the out of plane stress over the area of the model. An additional integral is now computed, which is the integral of the absolute value of the out of plane stress, and is used as the reference quantity for a given scalar out of plane strain. This accessed using the GeneralizedPlaneStrainReferenceResidual AuxKernel, and can be set up automatically by the Layered1DMaster Action.

\subsection{Capability to Group Variables}

For some cases, it is more appropriate to group several variables together to check convergence of their grouped solution rather than checking the convergence of the variables individually. For example, in a layered 1D solutions, there are multiple scalar variables that represent the out of plane strain, and because they have similar physical meaning, it makes sense to treat them as one vector, similar to a vector of degrees of freedom for a field variable.

When scalar variables are grouped together, the $L^{2}$ norm of the grouped residual for those variables is used to check convergence. The optional parameter group_variables is used to provide one or more lists of variable names which are to be grouped together. Multiple groupings of variables can be provided in group_variables by separating them 
by semicolon. Convergence for those variables that are not given in group_variables is checked individually. In an example, given below, displacements of disp_x and disp_y, and scalar variables of scalar_0, scalar_1 and scalar_2, are grouped together, respectively.

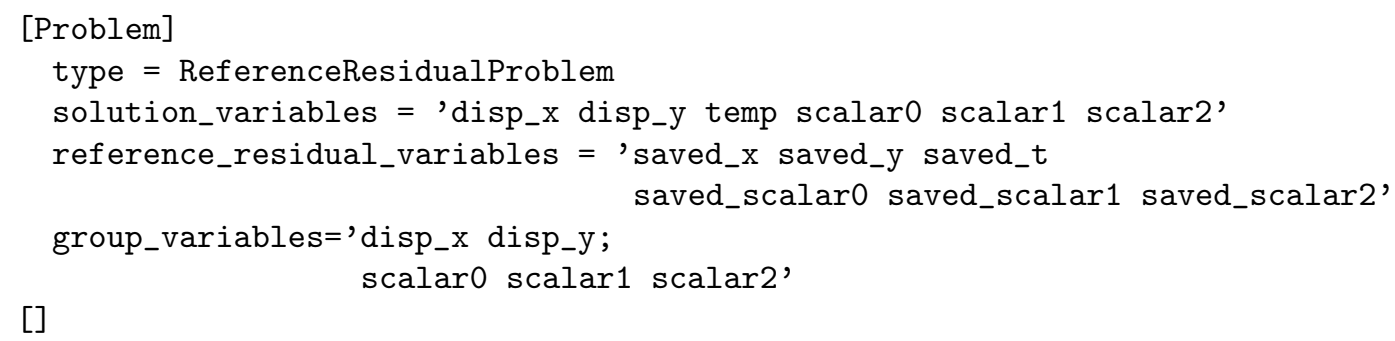

\subsection{Add and Group Scalar Variables in Layered1DMaster}

Layered 1D models consist of several 1D cylindrical fuel "slices" that each have up to two of their own out-of-plane scalar variables to represent the fuel and cladding for that layer if both are present on a given layer. We can use the Layered1DMaster action to create those scalar variables by specifying add_scalar_variables $=$ true. To be more user-friendly, we added new options in Layered1DMaster action to automatically add and group those scalar variables to ReferenceResidualProblem.

We use an existing example problem called Smeared_1.5D.i under BISON examples directory to demonstrate the new capability. Smeared_1.5D.i uses 10 equally sized axial slices to model a 10 pellet rodlet that has a total of 21 scalar variables to calculate the out of plane strain of fuel and cladding. The relevant input blocks are shown below. In the Problem block, only the standard variables and their reference residual variables are specified. In Layered1DMaster, parameter add_scalar_vars_to_reference_residual=true adds all scalars variables to ReferenceResidualProblem and parameter group_scalar_vars_in_reference_residual=true groups all the scalar variables together as one solution vector to check its convergence.

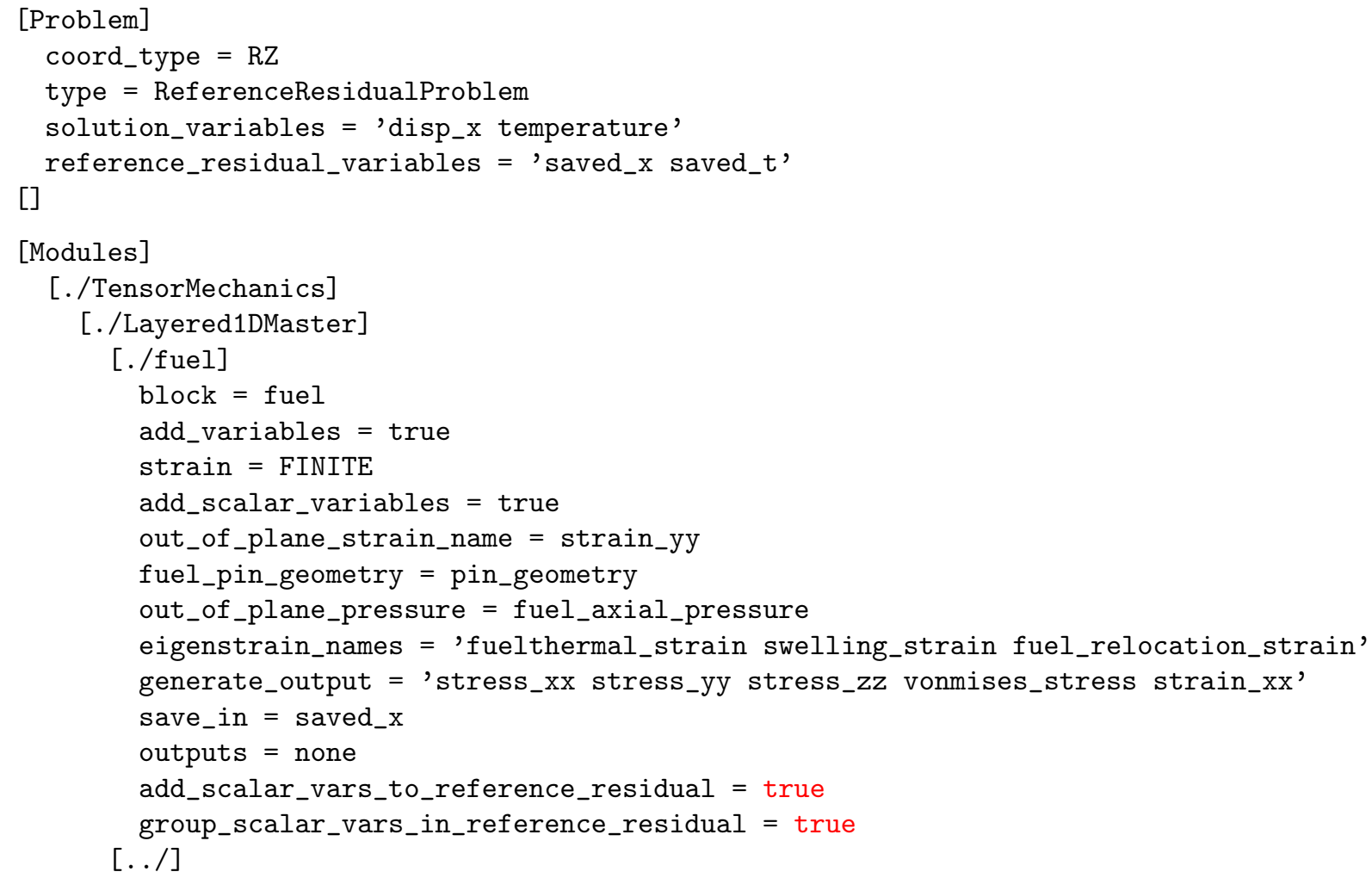




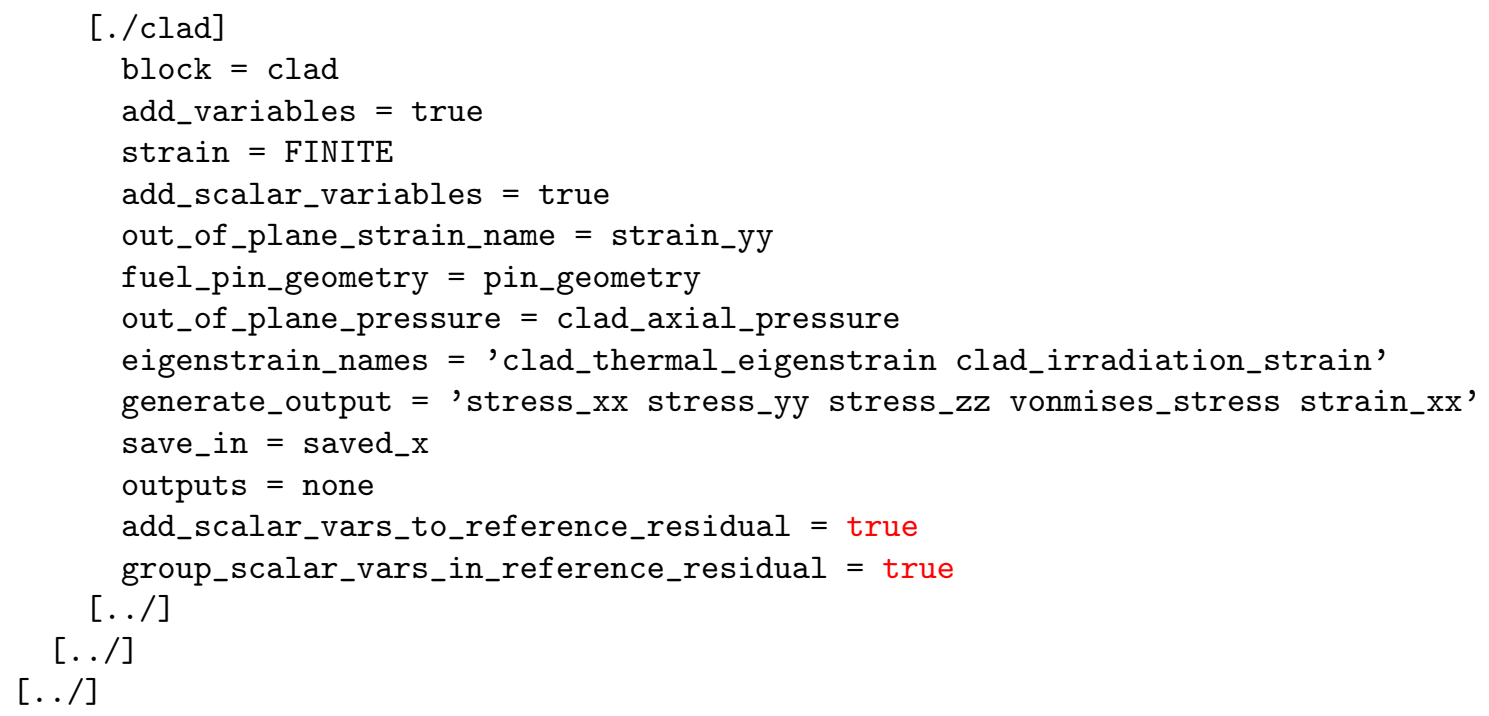

These options in the Layered1DMaster make the process of using ReferenceResidualProblem with layered 1D simulations much more straightforward, as they simplify the process of adding the potentially large list of scalar variables for the convergence check and group them together to check them in a way that is consistent with what is done for standard field variables. 


\section{Solver Option Investigation}

In an effort to have a basis to advise users on what solver option they should use, and to test the effectiveness of the new grouping option added to Bison, a small solution study was completed. The focus of this study was to investigate a range of solver tolerances and [Problem] block options with a Bison layered 1D input file. An input file representative of a CASL rod was selected for the study. The mesh has a total of 101 slices and is of a full length Pressurized Water Reactor (PWR) fuel rod. A set of cases were run and then outputs of interest, such as fuel centerline temperature (FCT) and fission gas release (FGR), were compared along with time to run. The tightest tolerance input file that would complete is used as the best answer for the other runs to compare against. Bison already recommends using the SuperLU solver within PETSc, so all runs were completed in this manner. The PETSc options used can be seen below, all runs were done serially and the only [Executioner] option changed between run sets was nl_rel_tol.

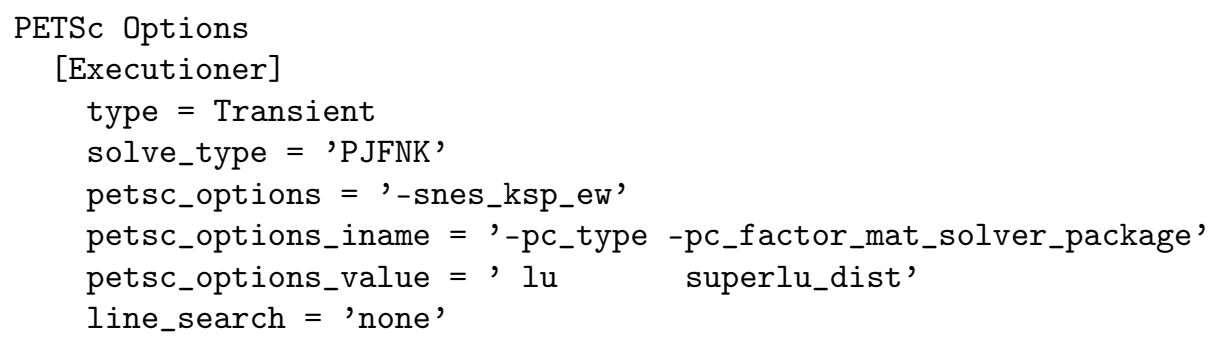

The only changes made to the run sets, as mentioned above, were changes to [Problem]. The first set were run with reference residual and the second set was run with reference residual and the new grouping option.

\subsection{Results}

Simulations in this study were attempted with nl_rel_tol values from 1e-1 to 1e-6. Tighter tolerances were not attempted due to previous findings in a similar study; the solves would not converge. Runtimes from simulations that completed are shown below in Table 1.

Results for a variety of standard outputs were compared in this study, but for the sake of brevity, only results from a small set of outputs of quantities of interest from these analyses with varying tolerances and grouping method are presented in Figures 1-6. In addition, plots of the per-step nonlinear iteration counts for the non-grouped and grouped cases are shown in Figures 7 and 8, respectively. Table 1 shows the overall simulation runtimes for these cases.

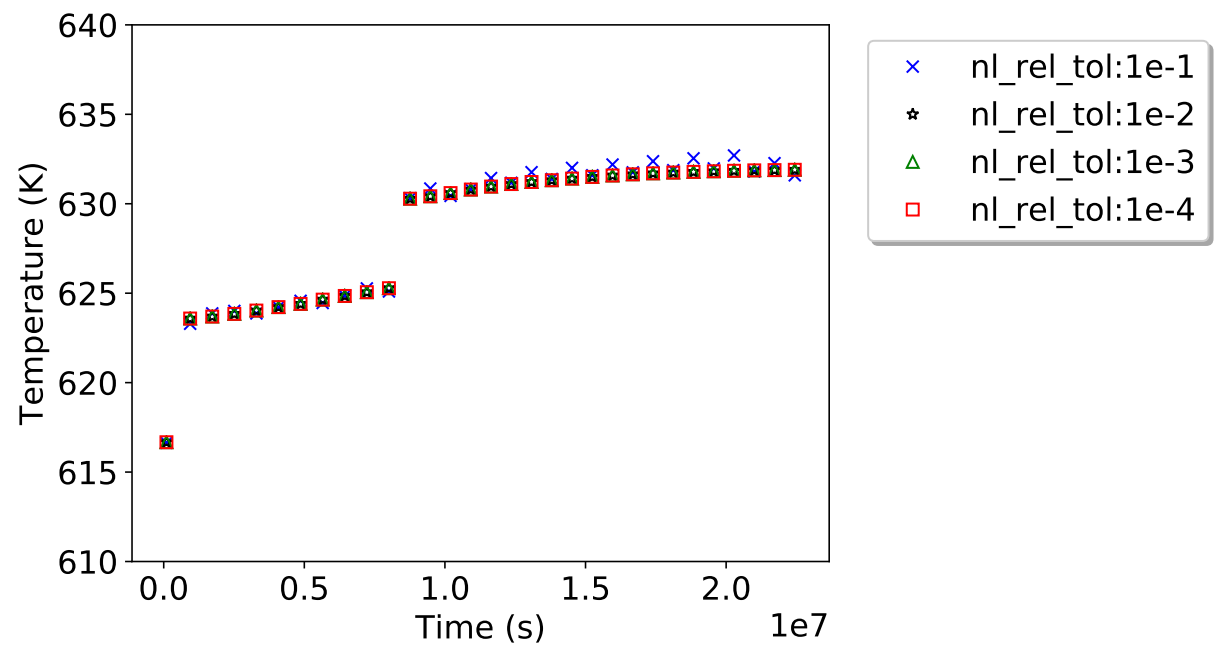

Figure 1: Bison calculated maximum cladding temperature for reference residual without grouping 


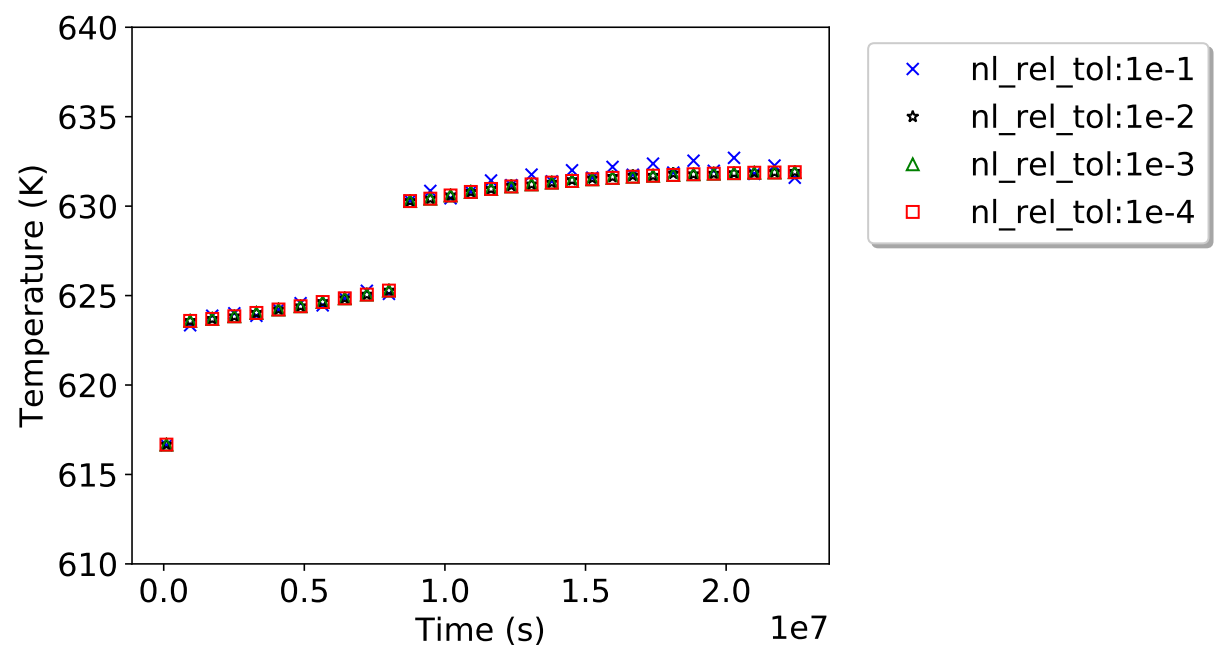

Figure 2: Bison calculated maximum cladding temperature for reference residual with grouping

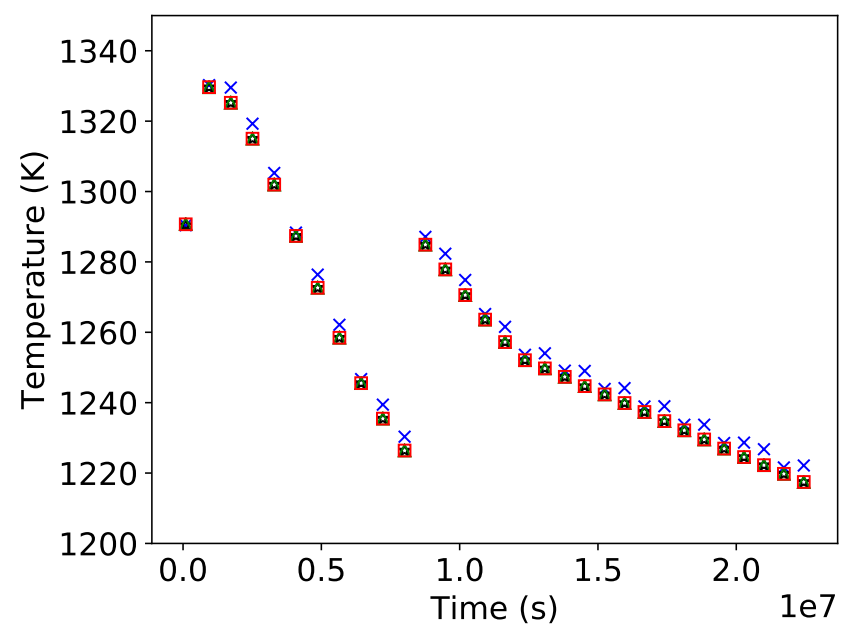

Figure 3: Bison calculated maximum fuel centerline temperature for reference residual without grouping

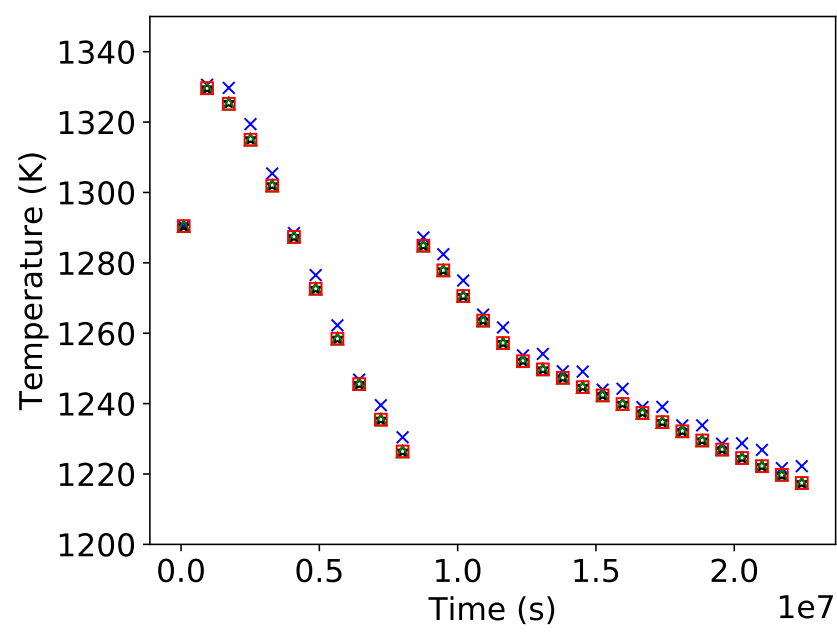

$\times \quad$ nl_rel_tol:1e-1

* nl_rel_tol:1e-2

$\Delta \quad$ nl_rel_tol:1e-3

- nl_rel_tol:1e-4

Figure 4: Bison calculated maximum fuel centerline temperature for reference residual with grouping 
All of the temperature based plots above are zoomed in over the temperature range to better show any result differences.

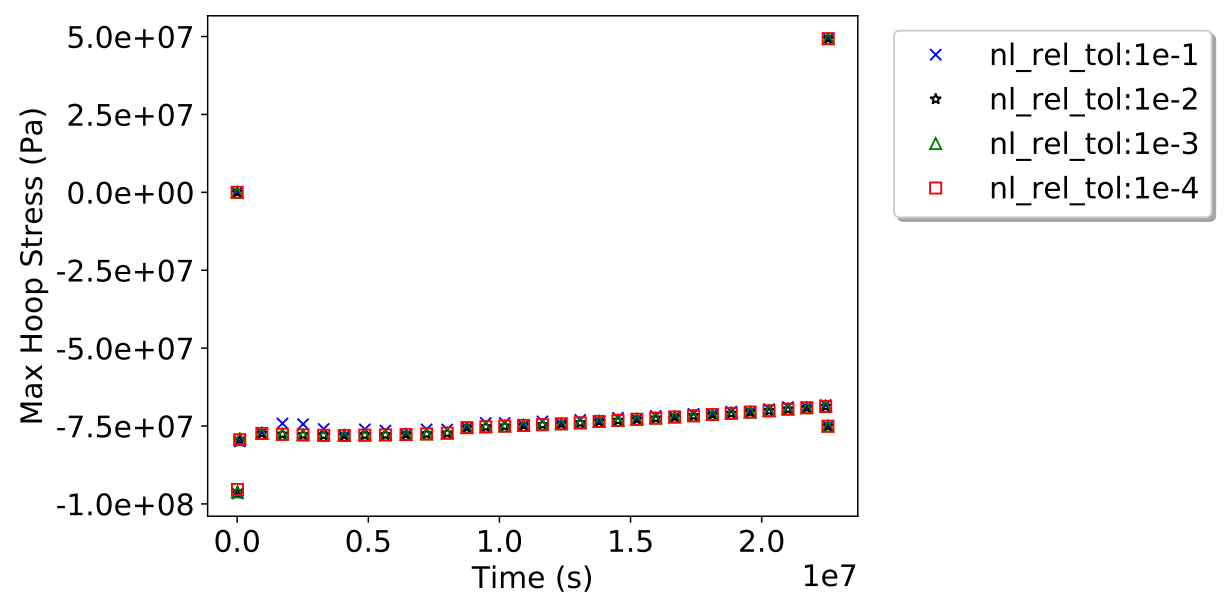

Figure 5: Bison calculated maximum cladding hoop stress for reference residual without grouping

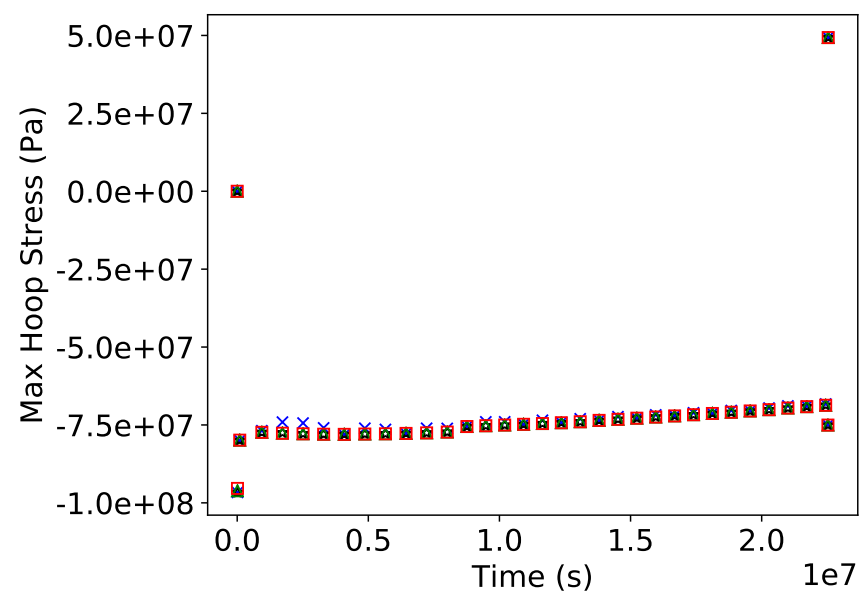

$\begin{array}{cc}\times & \text { nl_rel_tol: } 1 \mathrm{e}-1 \\ * & \text { nl_rel_tol:1e-2 } \\ \Delta & \text { nl_rel_tol:1e-3 } \\ \square & \text { nl_rel_tol: } 1 \mathrm{e}-4\end{array}$

Figure 6: Bison calculated maximum cladding hoop stress for reference residual with grouping 


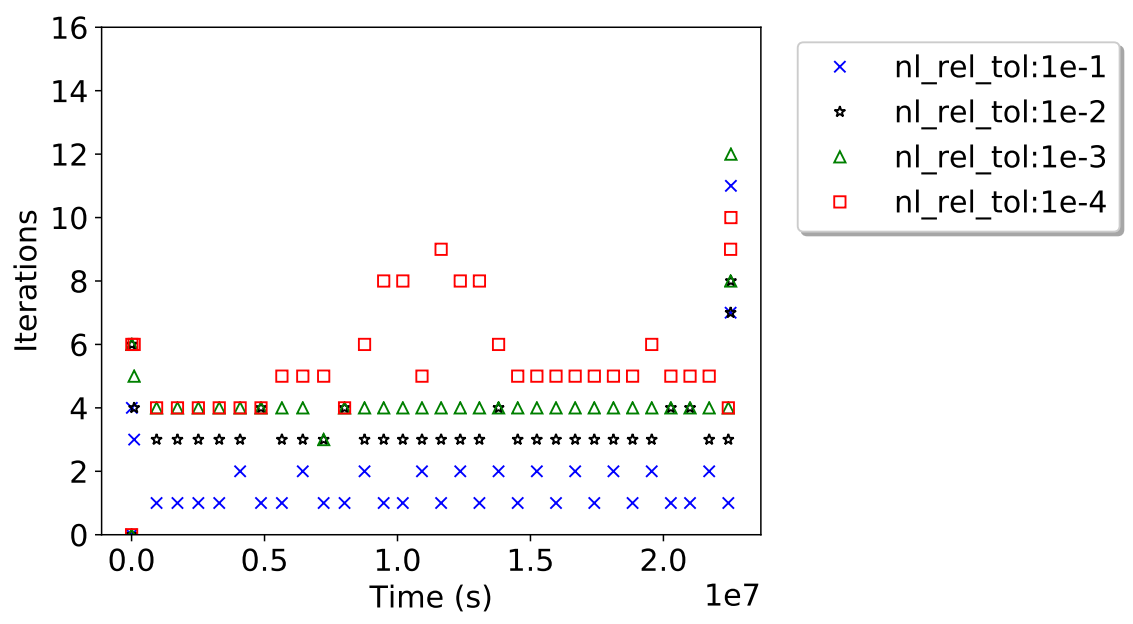

Figure 7: Nonlinear iteration count over time for reference residual without grouping

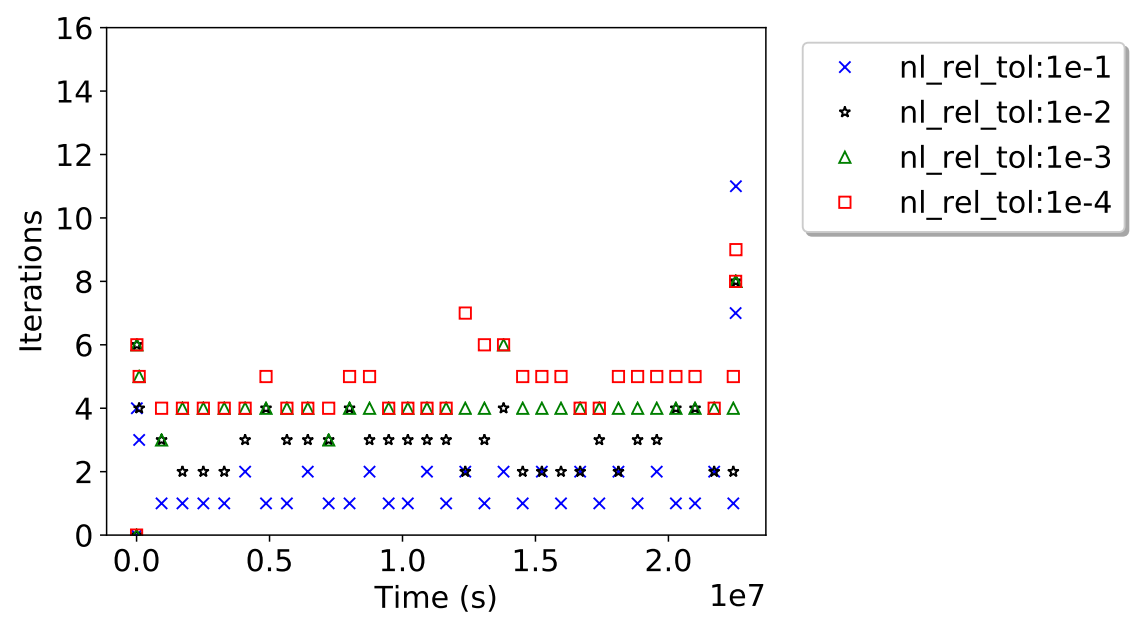

Figure 8: Nonlinear iteration count over time for reference residual with grouping 
Table 1: Summary of Simulation Runtimes with Varying Tolerances and Grouping Options

\begin{tabular}{|l|l|l|}
\hline Method & nl_rel_tol & Simulation Runtime (s) \\
\hline Reference residual without grouping & & \\
\hline & $1 \mathrm{e}-1$ & 172 \\
\hline & $1 \mathrm{e}-2$ & 315 \\
\hline & $1 \mathrm{e}-3$ & 450 \\
\hline & $1 \mathrm{e}-4$ & 802 \\
\hline Reference residual with grouping & & \\
\hline & $1 \mathrm{e}-1$ & 171 \\
\hline & $1 \mathrm{e}-2$ & 303 \\
\hline & $1 \mathrm{e}-3$ & 429 \\
\hline & $1 \mathrm{e}-4$ & 758 \\
\hline
\end{tabular}

\subsection{Conclusions}

From the results of a representative layered 1D analysis run with varying tolerances and with and without the grouping method, it can be seen that for both methods, when nl_rel_tol was varied from 1e-2 to 1e-4, the results are nearly indistinguishable. Only when this nonlinear relative tolerance is loosened to 1e-1 are significant differences noticeable in the quantities of interest that were tracked here. As would be expected, the required number of nonlinear iterations increases as the tolerance is tightened (decreased), and the simulation runtime increases accordingly. Also, the grouping method consistently requires a slightly smaller number of nonlinear iterations, which results in slightly lower runtimes. This is expected, as using the $L^{2}$ norm of the residual of the scalar variables is a slightly more permissive convergence criterion than is a requirement that every scalar variable meet the relative convergence criterion individually (infinity norm).

Based on these results, it is recommended that the grouping method be used, both because it is more consistent with the convergence checks used for the field variables and because of usability improvements (less verbose output). It is also clear that if nonlinear relative tolerance used with ReferenceResidualProblem is loser than 1e-2, solution accuracy will suffer in ways that are noticeable in results of engineering interest. Ideally, the tolerance should be set tighter than that when possible, although there are noticeable performance impacts in doing so. Studies such as this one should be performed on specific problems of interest to ensure that the tolerances used provide acceptable accuracy.

It is important to emphasize that these findings are only applicable when ReferenceResidualProblem is used. The standard MOOSE procedure computes convergence relative to the initial residual, which depends only on how far the solution is in the initial iteration from its converged state, and does not necessarily have any physical meaning. With ReferenceResidualProblem, convergence is computed relative to the applied boundary conditions, which is physically meaningful. 


\section{RIA Demonstration}

A demonstration of the CASL codes applied to a PWR control rod ejection accident (REA) for a 4-loop Westinghouse design typical of operating reactors with a typical core loading pattern was performed. The analysis consists of running CASL VERA, coupled Michigan Parallel Characteristics based Transport (MPACT) code [1] and COBRA-TF (CTF) [2], to simulate the PWR REA in whole core with pin-resolved transient neutronic using MPACT and core thermal-hydraulics modeled with CTF. The coupled VERA solution is then used as boundary conditions for a standalone Bison transient fuel rod thermal-mechanical analysis. The one-way coupling provides a higher-fidelity multiphysics analysis of the problem than each code individually. The complete details of this work are included in the report by Swindlehurst [3] but will be summarized here for completeness.

\subsection{VERA Model}

The Westinghouse 4-loop reactor design used for this demonstration analysis operates at $3459 \mathrm{MWt}$ with a typical cycle length of 500 EFPD. There are 193 fuel assemblies with a 17x17 fuel rod lattice. The analysis assumed operating conditions of flow $(136 \mathrm{Mlb} / \mathrm{hr})$, core inlet temperature $\left(290^{\circ} \mathrm{C}\right)$, and pressure $(15.5 \mathrm{MPa})$. The core design used in this demonstration analysis was selected because it has an atypical high ejected rod worth at hot zero power (HZP) and end-of-cycle (EOC) of \$1.20 [4]. The analysis is performed at EOC to obtain higher fuel assembly burnup as higher burnup fuel is of interest to reactivity-initiated accidents (RIA) because cladding performance is degraded.

The VERA core simulation model used in this analysis has the following characteristics.

- Full core geometry

- 60 axial meshes over full model height inclusive of nozzles

- MOC fuel mesh having 10 radial rings and 8 angular meshes

- Transport corrected P0 MOC scattering

- Epithermal up scatter model is disabled

- SP3 Nodal Axial Solver

The CTF subchannel simulation model used in this analysis has the following characteristics.

- CHF calculation is enabled allowing post DNB heat transfer regime

- Dynamic gap model is enabled

- Zero radial rings within the fuel pellet

- Burnup dependent fuel thermal conductivity is enabled

The transient VERA simulation is initiated from EOC HZP with the fully inserted control rod at core location D-12 ejected in 0.1 seconds. It was desired to increase the ejected rod worth to $\sim \$ 1.50$ for the purpose of obtaining a more severe power excursion. The core power transient response was increased by reducing core average beta-effective by a factor of 0.79 to 0.00422 . The reactivity excursion achieved $\$ 1.52$ at 0.125 seconds after the initiation of the rod ejection. The core average power climbed to $904 \%$ of full power at 0.170 seconds and the pulse had a full-width-athalf-max (FWHM) pulse of 31 msec.

Based on this core configuration, the fuel assembly in the ejected rod location (D-12) is a second-cycle assembly with an exposure of $41.9 \mathrm{GWd} / \mathrm{MTU}$. Following the ejection of the control rod the pin with the resulting highest power is located in the adjacent core location (D-13) with a first-cycle assembly with exposure of $23.7 \mathrm{GWd} / \mathrm{MTU}$. The peaking is higher in the adjacent assembly due to the lower assembly burnup and higher reactivity. The highest power rod in the highest power assembly (D-13-13-4) was chosen for the Bison analysis and for this rod the rod averaged power climbed to over $3500 \%$ of full power. The rod power history is shown in Figure 9. 


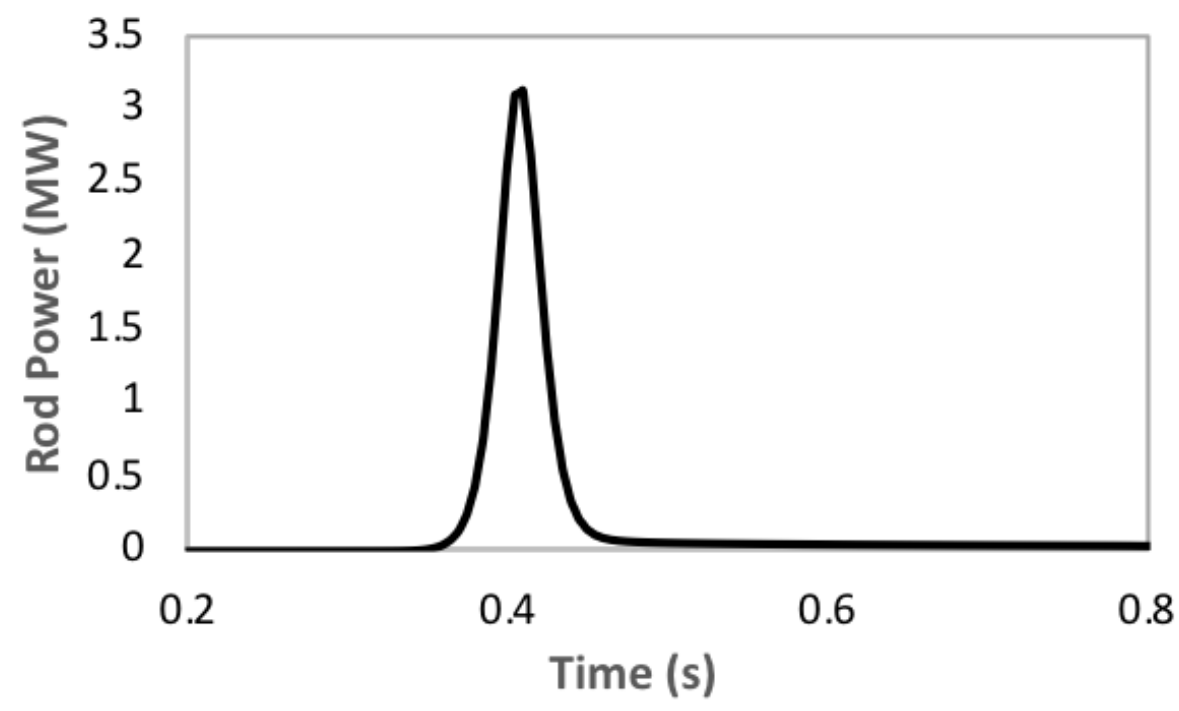

Figure 9: Rod D-13-13-4 rod power

\subsection{Bison Model}

Bison is used for a more detailed thermal-mechanical model of the fuel rod than the VERA code is capable of alone. The steps prior to the Bison analysis are as follows: First, the fuel rod design details that originate in the VERAIN database are used in the VERA analysis. Then VERA analyzes the coupled MPACT transient transport theory neutronics model with the CTF transient subchannel thermal-hydraulics model. The VERA rod ejection analyses results are then processed offline to convert the results into a BISON input file. The VERA outputs that are passed to BISON as transient boundary conditions are the axial power and the coolant channel temperature for a single fuel rod. BISON uses those boundary conditions to analyze the thermal-mechanical response of the fuel rod.

The Bison simulation begins as fresh fuel with the specified geometry and irradiation/thermal-hydraulic history from VERA and is run through its first cycle (in the D-13 assembly case) before the REA occurs at HZP conditions. The results of this irradiation history provide burnup-related fuel, gap, and cladding material properties and physical state such as the fuel radial power profile, fuel fission gas inventory, fission gas release affecting plenum gas pressure and composition, and cladding creep and oxidation. The Bison simulation is a 2D-RZ axisymmetric model (Figure 10) with 15 radial and 1000 axial cells for the full-length rod in the fuel and 4 radial and 1000 axial cells in the cladding. The radial mesh in the fuel is biased near the outer diameter to capture the rim region with higher power deposition at higher burnup. Details on the models and options for the Bison simulation are specified in Table 2.

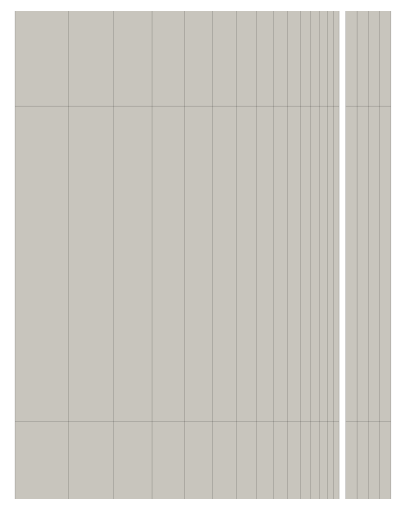

Figure 10: Bison 2D-RZ axisymmetric finite element mesh 
Table 2: Summary of Bison input models and parameters

\begin{tabular}{|l|l|}
\hline Model & Parameters \\
\hline Fuel thermal conductivity & NFIR \\
\hline Fuel mechanical model & $\begin{array}{l}\text { Elastic model with temperature } \\
\text { dependent properties }\end{array}$ \\
\hline Clad mechanical model & Creep and Plasticity models \\
\hline Clad material type & SRA Zry-4 \\
\hline Contact algorithm & Frictionless with Augmented Lagrange \\
\hline $\begin{array}{l}\text { Fission gas release and } \\
\text { swelling model }\end{array}$ & Sifgrs model \\
\hline Thermal hydraulic model & $\begin{array}{l}\text { Constant convection coefficient of } 40000 \\
\text { W/m } / \text { K with temporal and axial coolant } \\
\text { temperature from VERA }\end{array}$ \\
\hline Mechanical module & Tensor mechanics module \\
\hline Geometry & 2-D Radial axisymmetric \\
\hline $\begin{array}{l}\text { Mesh } \\
\text { Fuel } \\
\text { Clad }\end{array}$ & $\begin{array}{l}15 \text { (radial) x } 1000 \text { total per rod (axial) } \\
\text { (radial) }\end{array}$ \\
\hline Element Type & Quad-8 \\
\hline
\end{tabular}

\subsection{Bison Results}

The Bison simulation provides thermal results of higher fidelity compared to the VERA/CTF fuel rod model and include additional mechanical analysis results that are beyond the capabilities of the VERA/CTF model. The maximum fuel centerline temperature along with the rod power pulse from VERA are shown in Figure 11 and the fuel and cladding temperatures are shown in Figure 12. The temperature peaks at only $913 \mathrm{~K}$ which is less than the normal operating temperature of the fuel at full power. The reason for such a low fuel temperature even though the rod power is $3500 \%$ higher than full power is due to the short duration of the pulse. The total energy deposited in the fuel during the RIA is only $18 \mathrm{cal} / \mathrm{gUO} 2$ resulting in only an increase of $21 \mathrm{cal} / \mathrm{gUO} 2$ in the fuel radial average enthalpy from the HZP conditions with the maximum fuel radial average enthalpy of $45.9 \mathrm{cal} / \mathrm{UO} 2$ (Figure 13). This is a modest enthalpy increase relative to the magnitudes that have the potential to fail the cladding due to PCMI. The energy deposition assumed in this demonstration was moderate and there was no intent to challenge cladding failure.

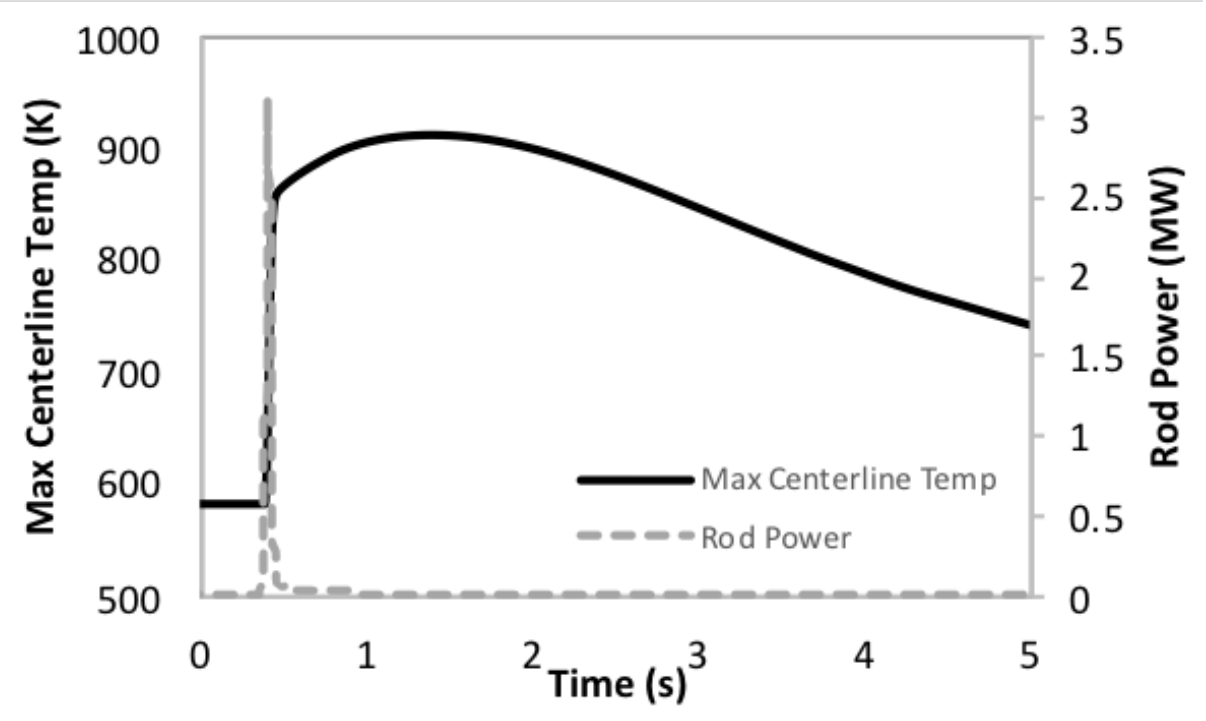

Figure 11: Bison calculated maximum fuel centerline temperature and VERA supplied rod power 


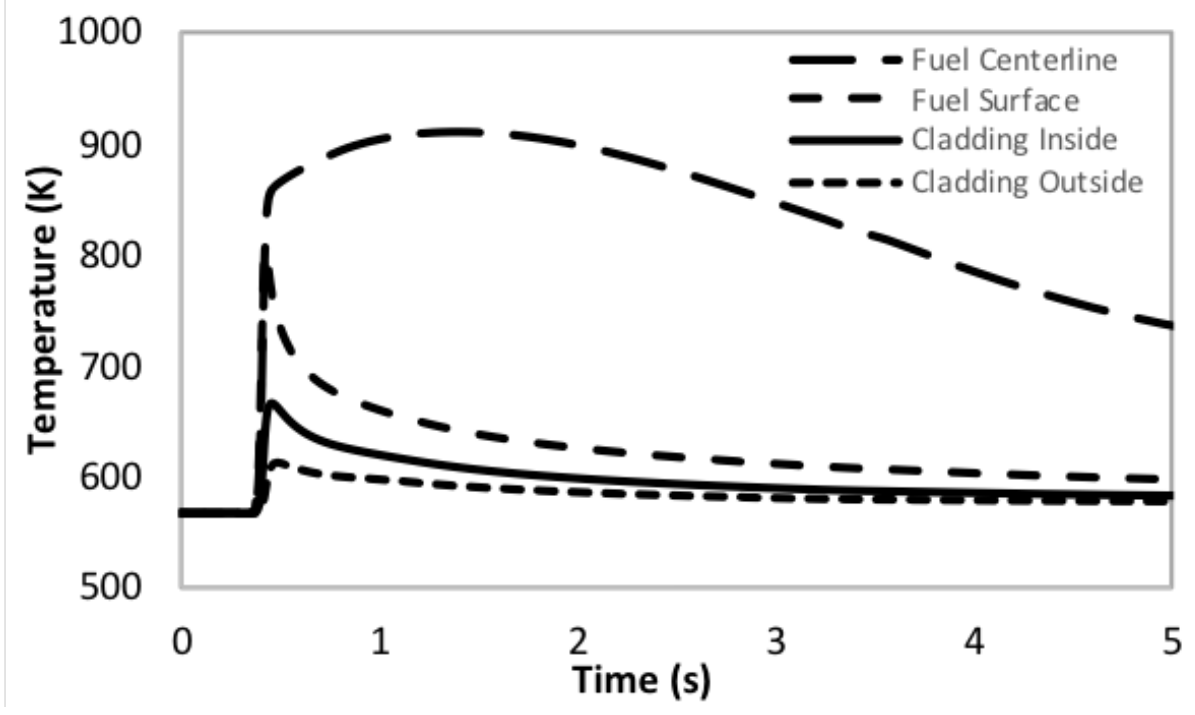

Figure 12: Bison calculated fuel and cladding temperatures

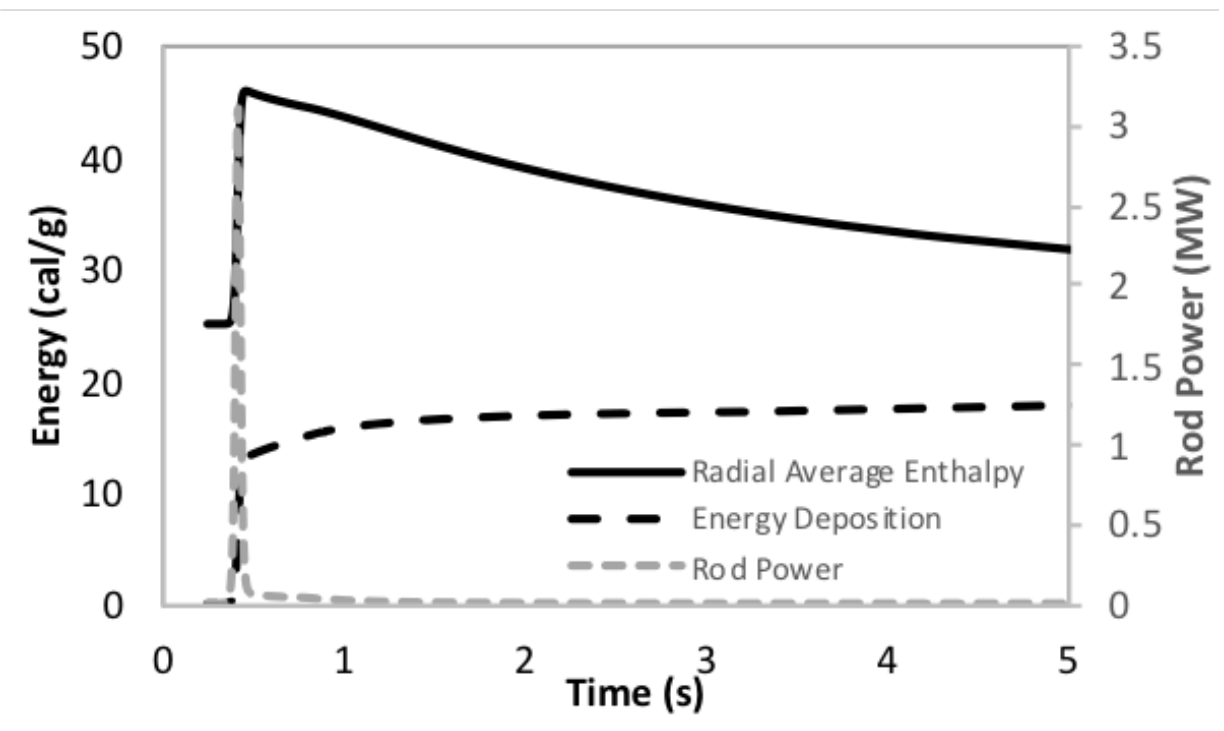

Figure 13: Bison calculated fuel radial average enthalpy and VERA supplied rod power and resulting energy deposition in fuel

Figure 14 shows the change in the fuel-to-cladding gap. For the initial condition of the analyzed fuel rod (e.g. burnup and power level) the gap is initially open. Then during the heatup and expansion of the fuel pellet during the power pulse, the gap width narrows but does not close. Figure 15 shows the response of the plenum pressure which is very similar to the external coolant pressure of 15.5 MPa. The heat transfer across the gap, along with the change in the gap volume due to thermal expansion, results in a small increase in plenum pressure. The cladding hoop stress is shown in Figure 16. The hoop stress is initially compressive due to the pressure difference across the cladding from the coolant to the gap. The power pulse results change the stress to a small tensile stress that quickly diminishes and returns to a compressive stress. The stress transient is small due to the gap remaining open and therefore no PCMI, and the small change in the pressure drop across the cladding. 


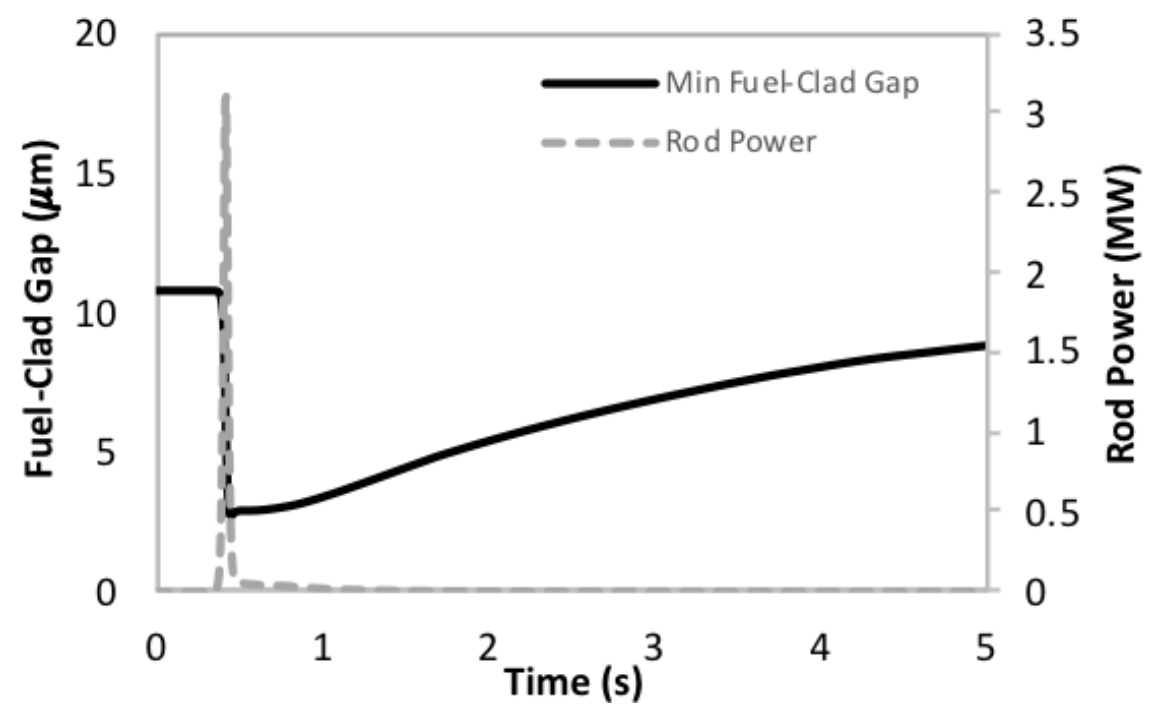

Figure 14: Bison calculated fuel-to-cladding gap width and VERA supplied rod power

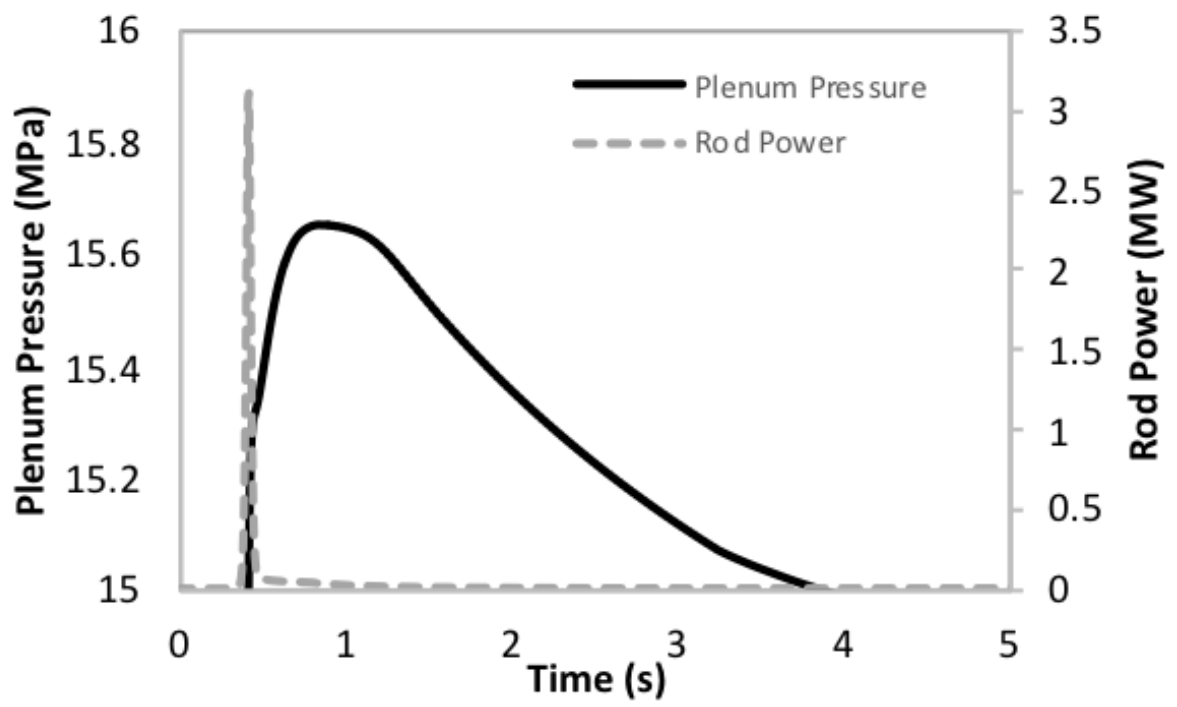

Figure 15: Bison calculated plenum gas pressure and VERA supplied rod power

\section{References}

[1] B. Collins and B. Kochunas. MPACT VERA common input user's manual. Technical Report CASL-U-20150043-000, February 2015.

[2] M. Avramova, A. Toptan, N. Porter, T. Blyth, C. Dances, A. Gomez, R. Salko, A. Wysocki, C. Jernigan, and J. Kelly. VERA 3.6 - CTF user's manual. Technical Report CASL-U-2017-1262-000, January 2017.

[3] G. Swindlehurst. Development, validation, and demonstration of CASL codes for the reactivity initiated accident. Technical Report CASL-U-2018-1646-000, August 2018.

[4] V. Kucukboyaci, B. Kochunas, T. Downar, A. Wysocki, and R. Salko. Evaluation of VERA-CS transient capability for analyzing the ap1000 reactor control rod ejection accident. In Physor 2018, April 22-26 2018. 


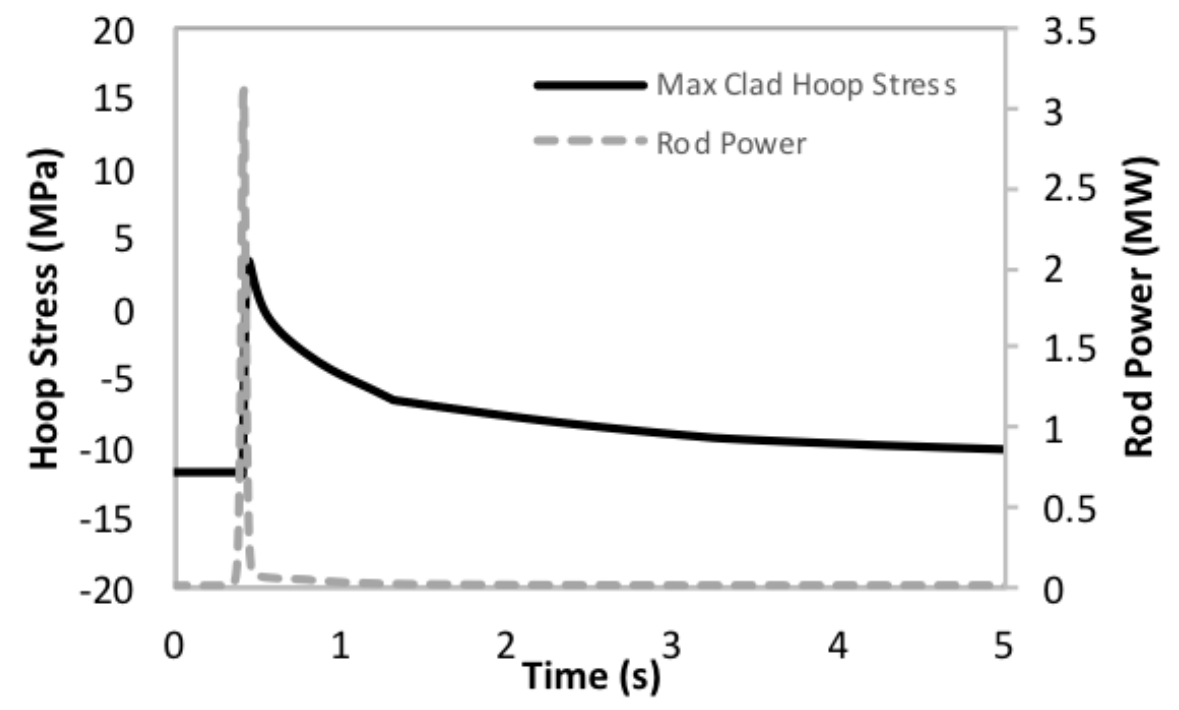

Figure 16: Bison calculated maximum cladding hoop stress and VERA supplied rod power 